\title{
Relativistic laser plasma interactions ${ }^{\star}$
}

\author{
Tünde Fülöp ${ }^{1}$, Francesco Pegoraro ${ }^{2}$, and Vladimir Tikhonchuk ${ }^{3, \text { a }}$ \\ ${ }^{1}$ Chalmers University of Technology, Göteborg, Sweden \\ ${ }^{2}$ University of Pisa, Pisa, Italy \\ ${ }^{3}$ University of Bordeaux, Bordeaux, France
}

Received 8 September 2017

Published online 23 November 2017 - (C) EDP Sciences, Società Italiana di Fisica, Springer-Verlag 2017

\begin{abstract}
High power laser physics takes a special place in the European landscape. The European roadmap programme Extreme Light Infrastructure with its three pillars in the Czech Republic, Hungary and Romania provides the international community with unique instruments, enabling access to relativistic plasmas in the laboratory. Such plasmas at the interface between laboratory size experiments and applications, extending from smallscale particle accelerators and brilliant photon sources to extreme astrophysical objects, need the development of new theoretical models, diagnostic tools and experimental methods combining relativistic plasma physics, quantum electrodynamics (QED) and nuclear physics.
\end{abstract}

This Topical Issue puts together the contributions of theoreticians from many countries. The core content of this Topical Issue is based on the papers presented at the First Chalmers Workshop on Relativistic Plasma Physics Theory which took place at Chalmers University of Technology in Göteborg, Sweden, October 5-7, 2016. The Workshop was focused on the issues related to the theory of relativistic laser pulse interactions with plasmas and applications to acceleration of electrons and ions to high energies. The scope of this Topical Issue extends to a broader domain including, besides relativistic plasma physics and QED, secondary sources of energetic particles and radiation, experiments on the interaction of relativistic laser pulses with solid targets, foams and gases, development of new numerical and diagnostic tools and applications of relativistic laser plasma interactions in nuclear physics, astrophysics and medicine.

There are 8 papers in this Topical Issue covering various theoretical and numerical subjects related to the interaction of relativistic laser pulses with plasmas and to applications to ion acceleration and hard X-ray emission. The papers by Magnusson et al. [1] and Fedeli et al. [2] consider ways of improving the absorption of

\footnotetext{
* Contribution to the Topical Issue "Relativistic Laser Plasma Interactions", edited by Tünde Fülöp, Francesco Pegoraro, Vladimir Tikhonchuk.

${ }^{a}$ e-mail: tikhonchuk@u-bordeaux.fr
}

intense laser pulses in structured targets and energy transfer to electrons. The authors demonstrate the possibility of controlling the electron energy and angular distribution and of enhancing the emission of high energy photons. Modelling of the laser interaction with sub-micron scale spikes or spheres is numerically challenging, but it opens up promising ways to create efficient secondary sources.

The papers by Wan et al. [3] and Popa et al. [4] analyze the efficiency of hard photon generation in the interaction of relativistic laser pulses with plasma electrons. By considering the electron radiation processes - bremsstrahlung emission of laser heated electrons and nonlinear Compton scattering, the authors show that the first process dominates for relatively low laser intensities below $10^{21}$ $10^{22} \mathrm{~W} / \mathrm{cm}^{2}$, while the second one becomes more important in the QED regime for intensities above $10^{22} \mathrm{~W} / \mathrm{cm}^{2}$.

The problems related to the optimization of ion acceleration with intense laser pulses are considered in the paper by Mackenroth et al. [5]. The authors address the practically important issues related to the optimization of the total number of accelerated ions and to their energy distribution by using a theoretical analysis and numerical simulations.

The authors of the next paper, Mendonça and Serbeto [6], propose an original concept of the electric charge of photon beams in a plasma. While some people might find such an approach counterintuitive, the editors believe that it might be useful for some applications and could stimulate interesting discussions.

Finally, the issues relating to numerical modeling of QED effects in plasmas are of prime importance for this new and growing domain of research. To some extent they are addressed in the majority of the papers presented in this issue, but for two of them the problem of the numerical solution of the relativistic Vlasov-Maxwell system is the major subject. The paper by Sarrat et al. [7] addresses the issue of an efficient parallelization of a semi-Lagrangian Vlasov-Maxwell solver by using a new algorithm of charge conservation. The paper by Wettervik et al. [8] addresses the problem of automatic mesh refinement, which is routinely used in hydrodynamic simulations but not yet in solving Maxwell's equations. The 
authors show the possibility of a significant improvement in the calculation speed while preserving good accuracy.

The editors believe that the proposed issue addresses the key problems of the physics of relativistic laser plasma interaction and will contribute to a better coordination of research in this domain in Europe.

\section{References}

1. J. Magnusson, A. Gonoskov, M. Marklund, Eur. Phys. J. D 71, 231 (2017)
2. L. Fedeli, A. Formenti, C.E. Bottani, M. Passoni, Eur. Phys. J. D 71, $202(2017)$

3. F. Wan, C. Lv, M. Jia, H. Sang, B. Xie, Eur. Phys. J. D 71, $236(2017)$

4. A. Popa, V. Stancalie, Eur. Phys. J. D 71, 166 (2017)

5. F. Mackenroth, A. Gonoskov, M. Marklund, Eur. Phys. J. D 71, 204 (2017)

6. J.T. Mendonça, A. Serbeto, Eur. Phys. J. D 71, 221 (2017)

7. M. Sarrat, A. Ghizzo, D. Del Sarto, L. Serrat, Eur. Phys. J. D 71, 271 (2017)

8. B.S. Wettervik, T.C. DuBois, E. Siminos, T. Fülöp, Eur. Phys. J. D 71, 157 (2017) 